\title{
Quantizer Design for Channel Codes With Soft-Output Decoding
}

\author{
Jan Bakus and Amir K. Khandani, Member, IEEE
}

\begin{abstract}
A new method of combined source-channel coding for the scalar quantization of a discrete memoryless source is presented, which takes advantage of the reliability information produced by a soft-output channel decoder. Numerical results are presented for a memoryless Gaussian source in conjunction with turbo code showing up to 1-dB improvement in the end-to-end distortion with respect to a traditional channel optimized scalar quantizer. The results include a Gaussian source designed using closed-form expression without the need for a training sequence, as well as image pixels using a training sequence. Furthermore, certain issues related to the effect of the channel mismatch and spectral efficiency of the system are studied. It is shown that the increase in distortion due to a channel mismatch can be substantially reduced by using an adaptive receiver.
\end{abstract}

Index Terms-Quantization, soft-output decoding, turbo code.

\section{INTRODUCTION}

$\mathbf{O}$ PTIMUM fixed-rate scalar quantizers, introduced by Max [1] and Lloyd [2], minimize the average distortion for a given number of threshold points. This approach was later extended by Linde et al. to vector quantizer [3]. The original Lloyd-Max algorithm does not consider the effect of channel noise. Kurtenbach and Wintz were among the first researchers who investigated the effect of the channel noise in a quantization system [4].

In [5], Farvardin and Vaishampayan presented an algorithm based on the Lloyd-Max algorithm for the quantizer design over a noisy channel resulting in the so called channel-optimized scalar quantizer (COSQ). The COSQ algorithm was further extended by the same researchers to vector sources in [6] and [7], which is known as the channel-optimized vector quantizer (COVQ).

One of the first attempts to design a quantizer with soft reconstruction decoding was made by Vaishampayan and Farvardin by extending the COVQ design algorithm to include the modulation signal set [8]. Another approach to soft reconstruction was proposed by Phamdo and Alajaji, who applied the COVQ algorithm to the case that the demodulator output is uniformly quantized to allow for a soft-decision decoding [9], [10]. This increases the number of channel output symbols and results in a finer reconstruction than the classical COVQ. This approach

Manuscript received June 10, 2002; revised November 24, 2003, July 17, 2004, and October 7, 2004. This work was supported in part by Communications and Technology Ontario (CITO). The review of this paper was coordinated by Prof. J. Shea.

J. Bakus is with Maplesoft, Waterloo, ON, N2V 1K8 Canada.

A. K. Khandani is with the University of Waterloo, Waterloo, ON, N2L 3G1 Canada.

Digital Object Identifier 10.1109/TVT.2004.841557 has successfully been used by Zhu and Alajaji [11] to design a COVQ for the turbo-code channel.

In [12] and [13], Skoglund and Hedelin study the vector quantization problem and present a soft decoder that is optimal in the mean-square sense and discuss rules to design the corresponding quantizer and reconstructor pair. This method is further adapted for image transmission [14], multiuser decoding [15], and channels with memory [16]. The algorithms based on Lloyd-Max quantizers are essentially gradient descent algorithms, which are not guaranteed to result in a global minimum. Some nondeterministic techniques have been proposed to deal with this; for example, deterministic annealing [17] and noisy channel relaxation [18]. An overview of such probabilistic techniques can be found in [19].

Ho [20] uses the reliability information and the same soft reconstruction rule as in this work. The design algorithm is based on the iterative COVQ and the effects of the channel noise are incorporated by transmitting the encoded training sequence through a turbo-code channel at every iteration. In our approach, we capture the effect of the turbo code in a model and design the quantizer using this model. Several other works have been devoted to using turbo code as part of a combined source-channel coding system for image transmission [21], [22] combining the JPEG standard [23] with channel coding by turbo code.

This work studies the transmission of a discrete time continuous amplitude signal over a noisy channel using a scalar quantizer in conjunction with a channel-coding scheme that relies on a soft-output channel decoder. ${ }^{1}$ An improvement in the end-to-end quantization distortion is achieved by providing a soft reconstruction rule using the reliability information generated by the soft-output channel decoder. The corresponding quantization and reconstruction rules are iteratively optimized using a procedure similar to the Lloyd-Max algorithm [1], [2].

The main contribution of this paper is to formulate these two basic principles explicitly for the case of a scalar quantizer and a channel decoder producing analog (unquantized) bit log likelihood ratio (LLR) values. The main differences between this paper and our earlier related work [26], [27] are: 1) improvement in the reconstruction level design by solving a system of linear equations and 2) using closed-form expressions to design the quantizers for the Gaussian distributed samples (instead of a training sequence).

This paper is organized as follows. Section II outlines the overall system including the design procedure for the quantizer and reconstructor. This is followed by several models for the

\footnotetext{
${ }^{1}$ A soft-output channel decoder can be efficiently implemented by applying the BCJR algorithm [24] to the trellis diagram of the code with iterations between decoders in the case of turbo code [25].
} 


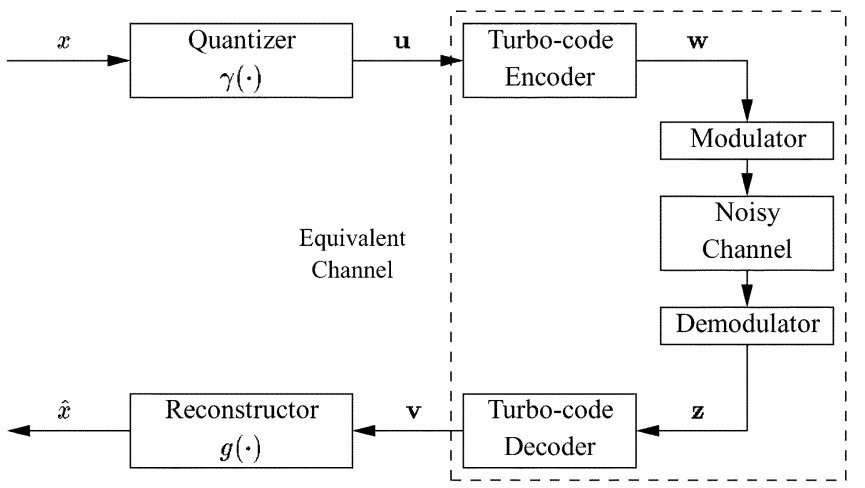

Fig. 1. System block diagram.

behavior of the channel-coding structure. Section III presents some numerical results for a memoryless Gaussian source in conjunction with turbo code. This includes a study of certain issues related to the effect of the channel mismatch and spectral efficiency of the system. Finally, the summary is given in Section IV.

\section{SYSTEM OVERVIEW}

The block diagram of the system is shown in Fig. 1. The scalar quantizer $\gamma(\cdot)$ maps the input sample $x$ to a quantizer partition $S \in\left\{S_{1}, \ldots, S_{N}\right\}$ and each quantizer partition is represented by a binary code word $\mathbf{u} \in\left\{\mathbf{u}_{1}, \ldots, \mathbf{u}_{N}\right\}$ composed of $r$ bits each, where $N=2^{r}$. Note that as the channel noise increases some of the quantizer partitions may be empty and, therefore, fewer than $N$ code words would be used. At the receiver side, for each transmitted code word $\mathbf{u}$, the soft-output decoder produces a reliability information vector $\mathbf{v}$ composed of $r \log$ likelihood ratio (LLR) values. The reconstructor $g(\cdot)$ maps the reliability information vector to an output sample $\hat{x}$.

For each bit $i$, the turbo decoder provides us with the LLR value $v_{i}$ defined as

$$
v_{i}=\ln \frac{P\left(u_{i}=1 \mid \text { channel output }\right)}{P\left(u_{i}=0 \mid \text { channel output }\right)}
$$

where the channel output is the entire received sequence for one block. The LLR value $v_{i}$ can be used to calculate the bit a posteriori probability according to [28]

$$
\begin{aligned}
& P\left(u_{i}=0 \mid v_{i}\right)=\frac{1}{1+\exp \left(v_{i}\right)} \\
& P\left(u_{i}=1 \mid v_{i}\right)=\frac{\exp \left(v_{i}\right)}{1+\exp \left(v_{i}\right)} .
\end{aligned}
$$

The encoder, noisy channel, and decoder can be considered as an equivalent channel with input $\mathbf{u}$ and output $\mathbf{v}$, as shown in Fig. 1. The turbo-decoding algorithm is based on bit interleaving, which results in a memoryless channel.
Given a source sample $x$ drawn from a source sample distribution $P(x)$ that is encoded in partition $i$ has the resulting distortion as

$$
D_{i}(x)=x^{2}-2 x B_{i}+C_{i}
$$

where

$$
\begin{aligned}
B_{i} & =\int_{\mathbf{v}} g(\mathbf{v}) P\left(\mathbf{v} \mid \mathbf{u}=\mathbf{u}_{i}\right) d \mathbf{v} \\
C_{i} & =\int_{\mathbf{v}} g(\mathbf{v})^{2} P\left(\mathbf{v} \mid \mathbf{u}=\mathbf{u}_{i}\right) d \mathbf{v} .
\end{aligned}
$$

The structure of the quantizer is captured in the quantities $B_{i}$ and $C_{i}$ given in (4). The optimal partition for the sample $x$ is given by

$$
S_{i}=\left\{x: D_{i}(x) \leq D_{j}(x), \forall j \neq i\right\} .
$$

The overall distortion for the entire system is given by

$$
D=\sum_{i=1}^{N} \int_{S_{i}} P(x)\left(x^{2}-2 x B_{i}+C_{i}\right) d x .
$$

We examine two approaches to reconstruct the sample. The output sample $\hat{x}$ is reconstructed with either hard reconstruction rule or soft reconstruction rule.

\section{A. Hard Reconstruction Rule}

The hard reconstruction rule selects the code word that is most likely transmitted and outputs the corresponding reconstruction level. Systems with this reconstruction rule are well established in the literature [1], [2], [4]-[7]. The hard noiseless channel [1], [2] model ignores the effect of the channel noise, such that the quantities $B_{i}$ and $C_{i}$ in (4) become

$$
\begin{aligned}
B_{i} & =R_{i} \\
C_{i} & =R_{i}^{2}
\end{aligned}
$$

and the reconstruction level is given by

$$
R_{m}=\frac{\int_{S_{m}} x P(x) d x}{\int_{S_{m}} P(x) d x}, \quad m=1, \ldots, N .
$$

The hard binary symmetric channel [5] models the channel as a binary symmetric channel with a crossover probability $\epsilon$. With this model, the quantities $B_{i}$ and $C_{i}$ in (4) become

$$
\begin{aligned}
B_{i} & =\sum_{j=1}^{N} P(j \mid i) R_{j} \\
C_{i} & =\sum_{j=1}^{N} P(j \mid i) R_{j}^{2}
\end{aligned}
$$

and the reconstruction level is given by

$$
R_{m}=\frac{\sum_{i=1}^{N} P(m \mid i) \int_{S_{i}} x P(x) d x}{\sum_{i=1}^{N} P(m \mid i) \int_{S_{i}} P(x) d x}, \quad m=1, \ldots, N .
$$


As the channel noise increases, one of the consequences is that not all partitions are used in the quantizer, which causes no problems in (10). However, since $P\left(\mathbf{u}=\mathbf{u}_{i} \mid \mathbf{v}\right)$ can be nonzero for the empty partitions, valid reconstructor levels are required for all partitions (including the empty ones).

\section{B. Soft Reconstruction Rule}

The soft reconstruction rule generates the reconstructed sample as a sum of the code word probabilities multiplied with their corresponding reconstruction levels, i.e.,

$$
\hat{x}=g(\mathbf{v})=\sum_{i=1}^{N} R_{i} P\left(\mathbf{u}=\mathbf{u}_{i} \mid \mathbf{v}\right)
$$

Using this soft-decision model, the parameters $B_{i}$ and $C_{i}$ are given by

$$
\begin{aligned}
B_{i} & =\sum_{k=1}^{N} R_{k} \Theta_{i}(k) \\
C_{i} & =\sum_{j=1}^{N} \sum_{k=1}^{N} R_{j} R_{k} \Psi_{i}(j, k)
\end{aligned}
$$

where

$$
\begin{aligned}
\Theta_{i}(k) & =\int_{\mathbf{v}} P\left(\mathbf{v} \mid \mathbf{u}=\mathbf{u}_{i}\right) P\left(\mathbf{u}=\mathbf{u}_{k} \mid \mathbf{v}\right) d \mathbf{v} \\
\Psi_{i}(j, k) & =\int_{\mathbf{v}} P\left(\mathbf{v} \mid \mathbf{u}=\mathbf{u}_{i}\right) P\left(\mathbf{u}=\mathbf{u}_{j} \mid \mathbf{v}\right) P\left(\mathbf{u}=\mathbf{u}_{k} \mid \mathbf{v}\right) d \mathbf{v} .
\end{aligned}
$$

The optimal reconstruction level corresponds to the minimum mean-square error (mmse) estimator $g(\mathbf{v})=E(x \mid \mathbf{v})$, which leads to

$$
R_{i}=E\left(x \mid x \in S_{i}\right)
$$

One of the characteristics of this design approach is that as the channel noise increases some of the partitions may be left empty, i.e., $\int_{S_{i}} P(x) d x=0$. As a consequence of this, the value $E\left(x \mid x \in S_{i}\right)$ is undefined for those empty partitions. One way to deal with this is to assign the value $E(x)$ to the empty partitions, resulting in

$$
R_{i}= \begin{cases}E\left(x \mid x \in S_{i}\right), & \int_{S_{i}} P(x) d x>0 \\ E(x), & \int_{S_{i}} P(x) d x=0\end{cases}
$$

We refer to this case as the expected value, which was presented in [26].

In this work, we present a different approach to deal with the empty partitions that is based on minimizing the average distortion in (6) through setting its derivative with respect to $R_{m}, m=1, \ldots, N$, equal to zero. This results in $N$ equations in terms of the $N$ reconstruction levels

$$
2 \sum_{i=1}^{N} \int_{S_{i}} x P(x) d x \frac{d B_{i}}{d R_{m}}=\sum_{i=1}^{N} \int_{S_{i}} P(x) d x \frac{d C_{i}}{d R_{m}}
$$

where $m=1, \ldots, N$. By substituting $B_{i}$ and $C_{i}$ from (12), we obtain a set of linear equations

$$
\begin{aligned}
\sum_{i=1}^{N} \Theta_{i}(k) \int_{S_{i}} x P(x) d x= & \sum_{j=1}^{N} R_{j} \\
& \times \sum_{i=1}^{N} \Psi_{i}(j, k) \int_{S_{i}} P(x) d x
\end{aligned}
$$

where $k=1, \ldots, N$. This set of linear equations can be expressed in matrix form as

$$
\left[\begin{array}{c}
E(1) \\
\vdots \\
E(N)
\end{array}\right]=\left[\begin{array}{ccc}
F(1,1) & \cdots & F(1, N) \\
\vdots & \ddots & \vdots \\
F(N, 1) & \cdots & F(N, N)
\end{array}\right]\left[\begin{array}{c}
R_{1} \\
\vdots \\
R_{N}
\end{array}\right]
$$

where

$$
\begin{gathered}
E(k)=\sum_{i=1}^{N} \Theta_{i}(k) \int_{S_{i}} x P(x) d x \\
F(j, k)=\sum_{i=1}^{N} \Psi_{i}(j, k) \int_{S_{i}} P(x) d x .
\end{gathered}
$$

The solutions $R_{i}, i=1, \ldots, N$ to this set of equations result in the optimal reconstruction levels. Note that, in the case of no empty partitions, i.e., $\forall i, \int_{S_{i}} P(x) d x>0$, this system of equations is equivalent to the expected value case in (15). We refer to this case as the system of equations and we show numerically that, in the case of empty partitions, it performs better than the expected value case. The derivation of this system is presented in the Appendix.

Note that the existence of empty partitions cannot be captured in our channel model because the basic assumption is that the conditional symbol probabilities are computed by multiplying the conditional probabilities of their bit components. This is a crucial ingredient of the turbo-decoding algorithms based on bit interleaving. This model "cannot capture" the effect of the empty partitions because the probabilities of the symbols (computed by multiplying the probabilities of their bit components) will be always nonzero. In other words, using the bit independence assumption and (2), one obtains a nonzero probability $P\left(\mathbf{u}=\mathbf{u}_{i} \mid \mathbf{v}\right)$ even for an empty partition $S_{i}$. The presented formulation accounts for this effect in a proper manner.

\section{Channel-Parameters Estimation}

The two sets of parameters, $\Theta_{i}(k)$ and $\Psi_{i}(j, k)$, capture the characteristics of the channel with soft reconstruction rule. The indexes $i, j$ and $k$ denote the individual code words and $\Theta_{i}(k), \Psi_{i}(j, k)$ are computed for all possible code word combinations. To calculate these parameters, we first compute the single bit versions $\theta_{i}(k)$ and $\psi_{i}(j, k)$ where

$$
\begin{aligned}
\theta_{i}(k) & =\int_{v} P(v \mid u=i) P(u=k \mid v) d v \\
\psi_{i}(j, k) & =\int_{v} P(v \mid u=i) P(u=j \mid v) P(u=k \mid v) d v .
\end{aligned}
$$


The indexes $i, j$, and $k$ in (20) can take the binary values 0 or 1. The two quantities are evaluated for all possible bit combinations, resulting in four different values for $\theta_{i}(k)$ and eight different values for $\psi_{i}(j, k)$. Using the independence assumptions, the set of parameters $\Theta_{i}(k)$ and $\Psi_{i}(j, k)$ are calculated by multiplying their single bit components, i.e., $\theta_{i}(k)$ and $\psi_{i}(j, k)$.

In order to estimate the channel parameters $\theta_{i}(j)$ and $\psi_{i}(j, k)$, the equivalent channel in Fig. 1 is replaced by a reliability information channel model. This channel is based on the assumption that the equivalent channel model outputs a reliability information value $P\left(u_{i}=k \mid v_{i}\right)$ for each input bit $u_{i}$, where $k \in\{0,1\}$. We define the reliability information variables $x_{0}$ and $x_{1}$ as

$$
\begin{aligned}
& x_{0}=P(u=0 \mid v) \\
& x_{1}=P(u=1 \mid v) .
\end{aligned}
$$

At each time step, the values $x_{0}$ and $x_{1}$ are different depending on the channel noise, transmitted bit, and the decoding algorithm and, therefore, we can treat them as random variables with a probability density function (pdf) of $P\left(x_{0}\right)$ and $P\left(x_{1}\right)$, respectively. Equation (2) is functions that map $v$ to $x_{0}$ and $x_{1}$ as

$$
\begin{aligned}
& x_{0}=\frac{1}{1+\exp (v)} \\
& x_{1}=\frac{\exp (v)}{1+\exp (v)} .
\end{aligned}
$$

Since these two functions are strictly monotone, we can define the relationship between the pdf of $v$ and the pdf of $x_{0}$ and $x_{1}$.

$$
P\left(x_{k}\right) d x_{k}=P(v) d v, \quad k=0,1
$$

Conditioning both sides of the equation on $u=i$ results in

$$
P\left(x_{k} \mid u=i\right) d x_{k}=P(v \mid u=i) d v .
$$

Substituting (21), (24) in (20) results in

$$
\begin{aligned}
\theta_{i}(k) & =\int_{v} P(v \mid u=i) P(u=k \mid v) d v \\
& =\int_{0}^{1} x_{k} P\left(x_{k} \mid u=i\right) d x_{k} \\
& =E\left(x_{k} \mid u=i\right) .
\end{aligned}
$$

The quantity $E\left(x_{k} \mid u=i\right)$ is measured by passing a test bit stream through the channel and observing the channel output.

The quantity $\psi_{i}(j, k)$ is calculated by defining four random variables $y_{j, k}$ as

$$
y_{j, k}=P(u=j \mid v) P(u=k \mid v), \quad j, k=0,1 .
$$

Similarly, we define the relationship between the pdf of $v$ and the pdf of $y_{j, k}$ as

$$
P\left(y_{j, k}\right) d y_{j, k}=P(v) d v
$$

Conditioning both sides on $u=i$ results in

$$
P\left(y_{j, k} \mid u=i\right) d y_{j, k}=P(v \mid u=i) d v \text {. }
$$

Finally, substituting (26), (28) in (20) results in

$$
\begin{aligned}
\psi_{i}(j, k) & =\int_{v} P(v \mid u=i) P(u=j \mid v) P(u=k \mid v) d v \\
& =\int_{0}^{1} y_{j, k} P\left(y_{j, k} \mid u=i\right) d y_{j, k} \\
& =E\left(y_{j, k} \mid u=i\right) .
\end{aligned}
$$

The quantity $E\left(y_{j, k} \mid u=i\right)$ is also measured by passing a test bit stream through the channel and observing the output. Using the parameters $\theta_{i}(j)$ and $\psi_{i}(j, k)$, we calculate the parameters $\Theta_{i}(j)$ and $\Psi_{i}(j, k)$ and design the quantizer as outlined before.

\section{NUMERICAL RESULTS}

\section{A. Simulation Setup}

The proposed quantization scheme has been simulated for an independent and identically distributed (i.i.d.) Gaussian input. The channel is additive white Gaussian noise (AWGN) with binary phase-shift keying (BPSK) modulation. ${ }^{2}$ The quantizers are designed and tested with a turbo code with a block length of 10000 bits. The turbo codes presented use an $S$-random interleaver [29] with $S=15$ and the number of iterations for the turbo decoder is equal to 5 . The main simulation is performed using a rate $1 / 3$ turbo code, composed of two component codes with $M=2$ memory elements, forward polynomial (101) and backward polynomial (111). The BER curve for this code are shown in Fig. 2(a).

To show the tradeoff between the source and channel coding, some simulations are also performed with rates $1 / 2,1 / 3$, and $1 / 4$ turbo code. The turbo code used for these simulations has $M=3$ memory elements and block length of 10000 bits. To generate the different rates, the basic turbo-code structure has been modified by replacing each of the component codes with a pair of encoders. The two encoders in each pair have the forward polynomials (1011), (1111) and the same backward polynomial (1101), as well as different coding rates achieved by puncturing the encoder outputs. The BER curves for these codes using a block length of 10000 are shown in Fig. 2(b).

The total of four different quantizers are designed and tested, as listed in Table I. The first quantizer is the classical LloydMax quantizer designed assuming noiseless channel and tested over a noisy channel with hard decision rule. The channel-optimized quantizer is designed for the noisy channel and tested with a hard decision rule. Two soft-decision rule quantizers are designed using the reliability information channel. The first is designed using the expected value in (33) to calculate the reconstruction levels (where the undefined reconstruction levels

\footnotetext{
${ }^{2}$ The specific model of AWGN channel with BPSK modulation is used as a framework to present numerical results. However, the quantization methods proposed are general and can be used in conjunction with other channel models and/or modulation schemes.
} 


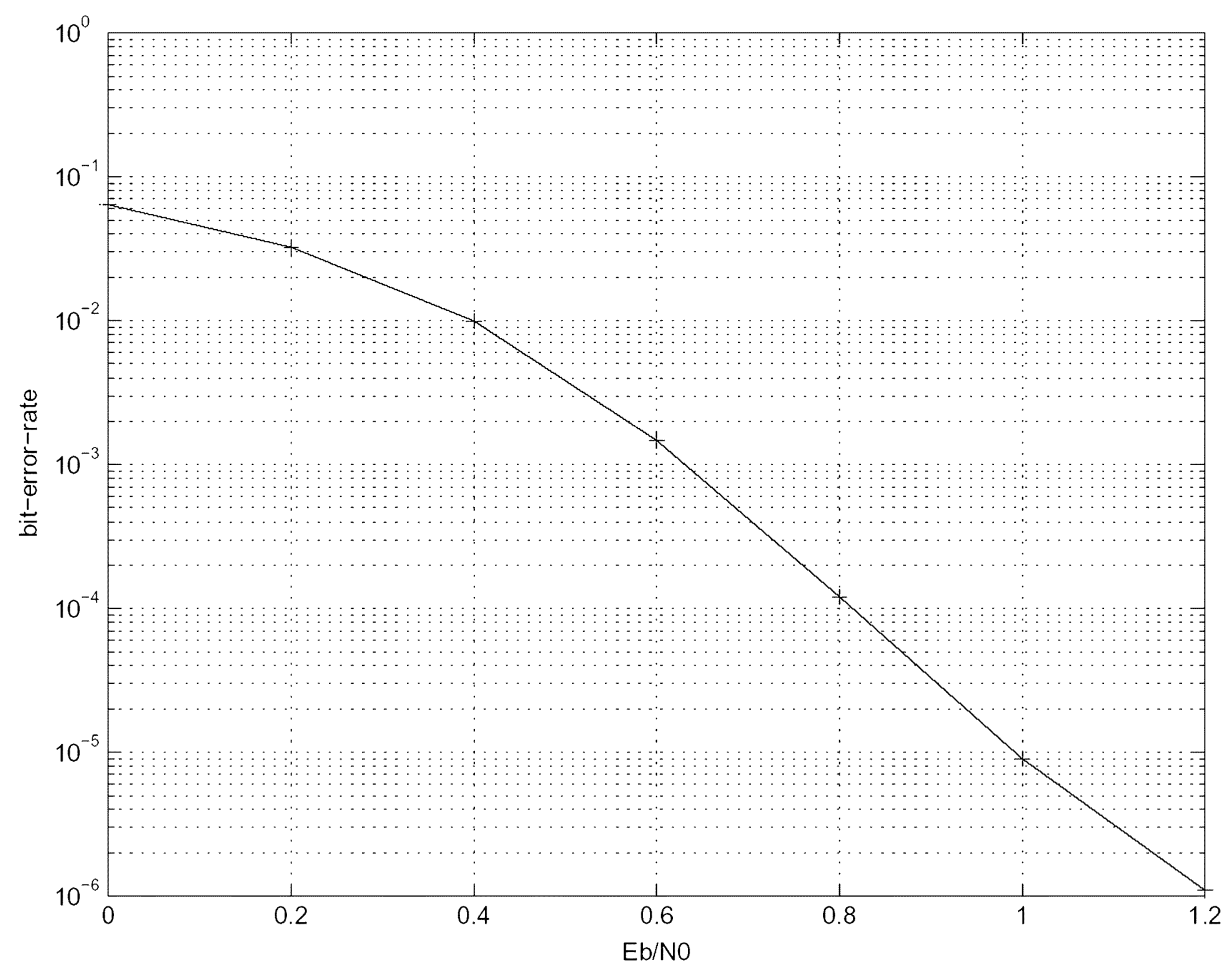

(a)

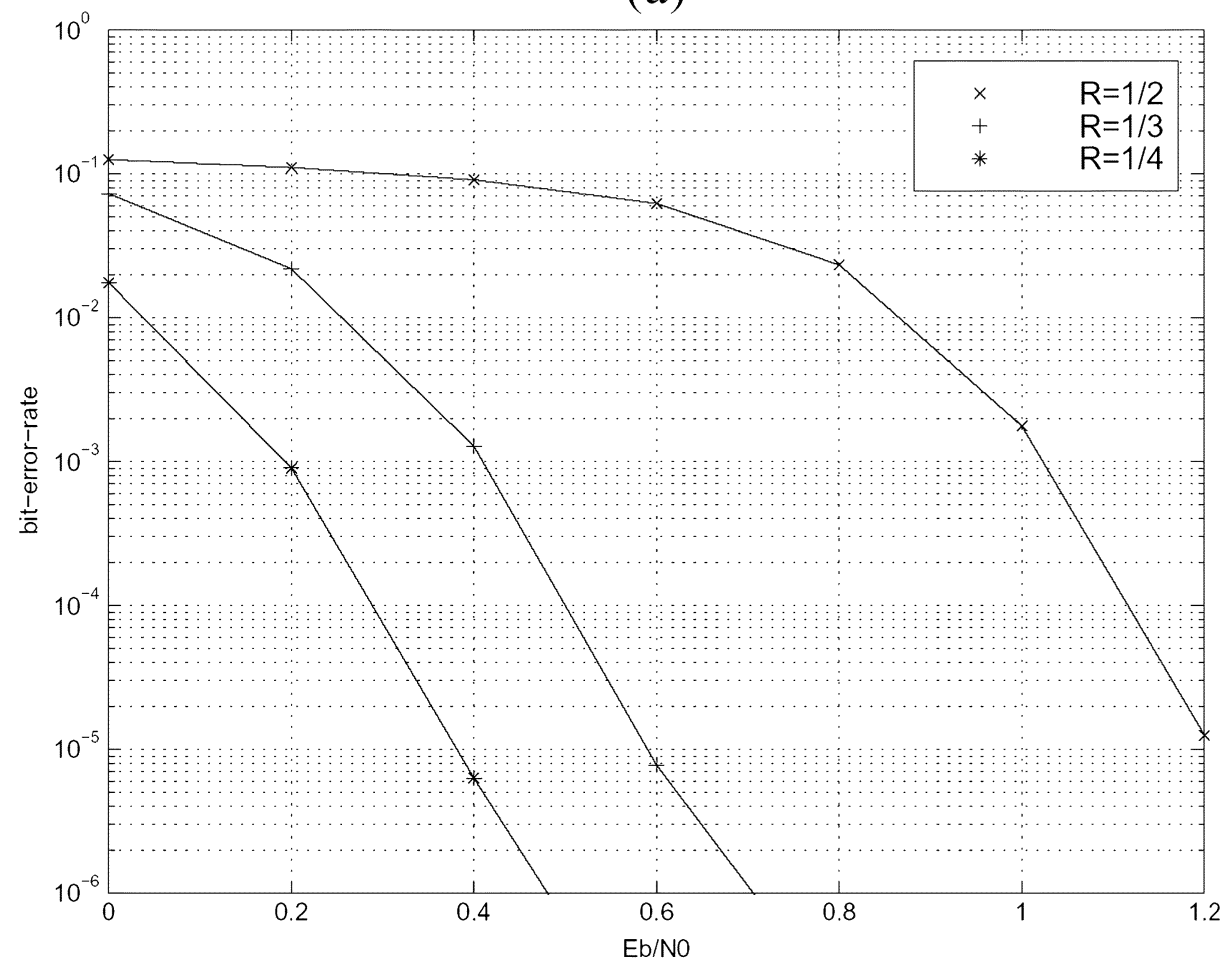

(b)

Fig. 2. BER curves for turbo-code channel coding. (a) $M=2$, rate $1 / 3, N=10000$. (b) $M=3, N=10000$.

TABLE I

QUANTIZERS TESTED

\begin{tabular}{l|l}
\hline Quantizer & Channel Model \\
\hline Lloyd-Max & Noiseless \\
Channel Optimized & Binary Symmetric \\
Soft Decision (Expected Value) & Reliability Information \\
Soft Decision (System of Equations) & Reliability Information \\
\hline
\end{tabular}

are selected as the mean of the source sample distribution), while the second one uses the system of equations in (17).

To reduce the impact of the initialization, the code words are initialized randomly and the best of 100 designs is chosen. In each case, the quantizer and reconstructor design algorithms are iterated until the relative change in the mean-square error (mse) 


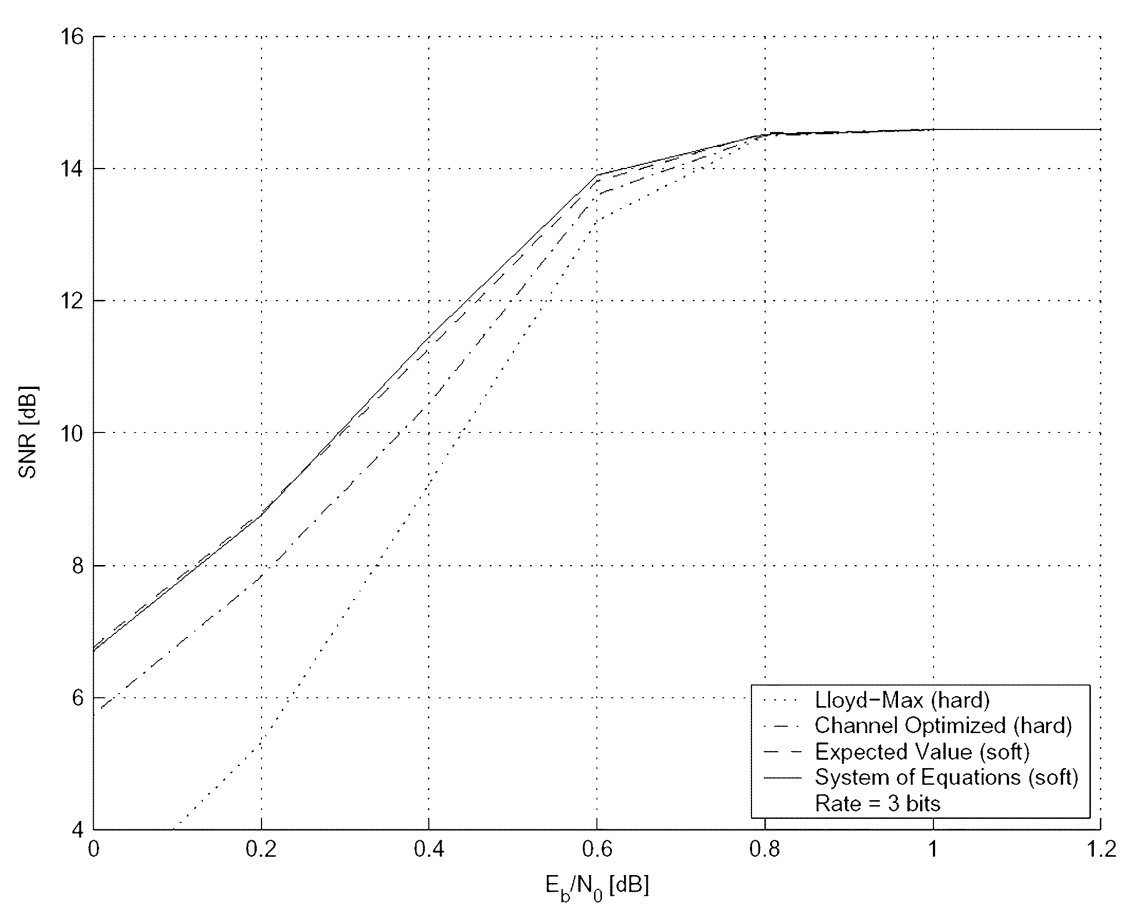

(a)

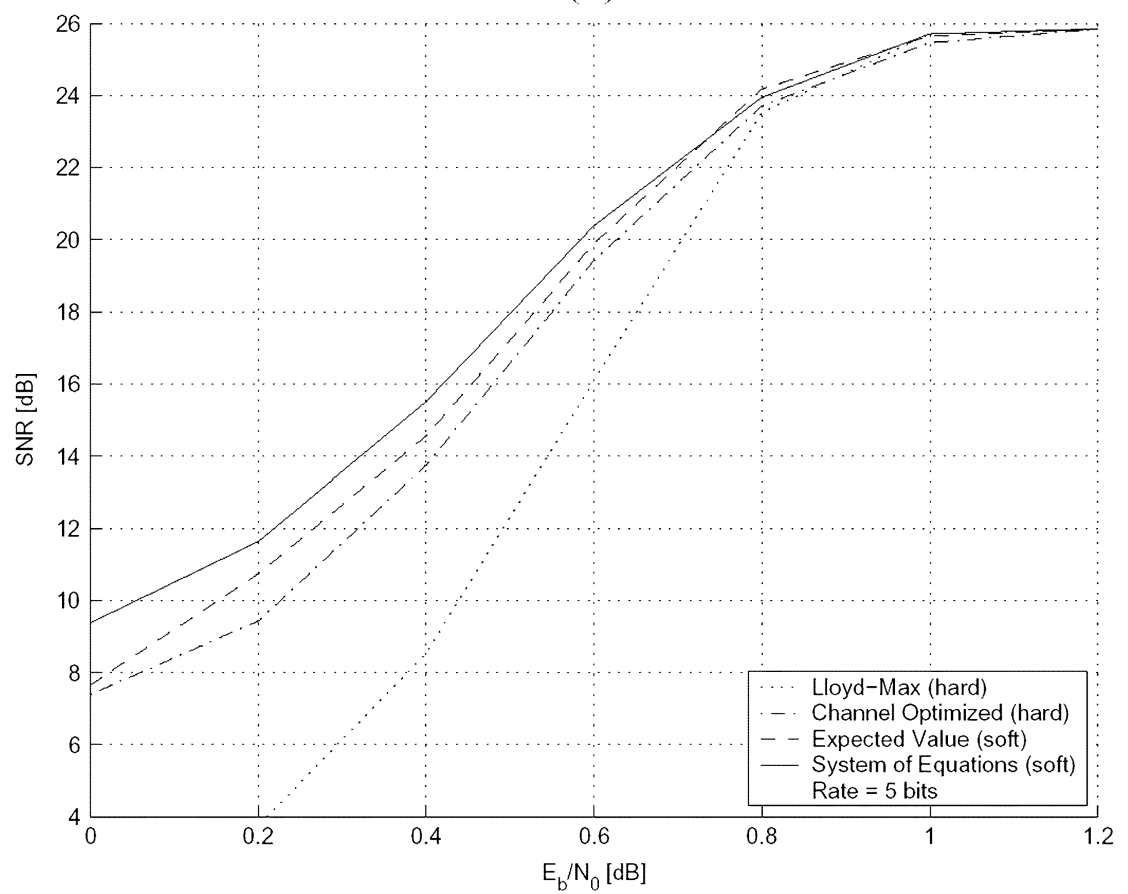

(b)

Fig. 3. SNR (SNR) for 3- and 5-bit quantizers with rate $1 / 3$ turbo code with $M=2$ memory elements. (a) Three-bit quantizers. (b) Five-bit quantizers.

is less that $10^{-3}$. Since the source sample distribution is known to be Gaussian, the quantizers are designed with the closed-form expression without the use of a training sequence.

\section{B. Quantizer Results}

The results for 3- and 5-bit quantizers are shown in Fig. 3. As expected, the Lloyd-Max quantizer operating with hard reconstruction rule results in the worst performance. The channel-optimized quantizer also uses a hard reconstruction rule; how- ever, the design takes the effect of the channel noise into account and the performance is substantially improved. The soft reconstruction quantizers offer an improvement over both of the hard reconstruction quantizers. Of the two soft-decision quantizers, the system of equations design performs better than the expected value design. Fig. 4 shows the effect of using soft reconstruction rule with the Lloyd-Max and channel-optimized quantizers, where the performance of both quantizers is improved using the soft reconstruction rule. 


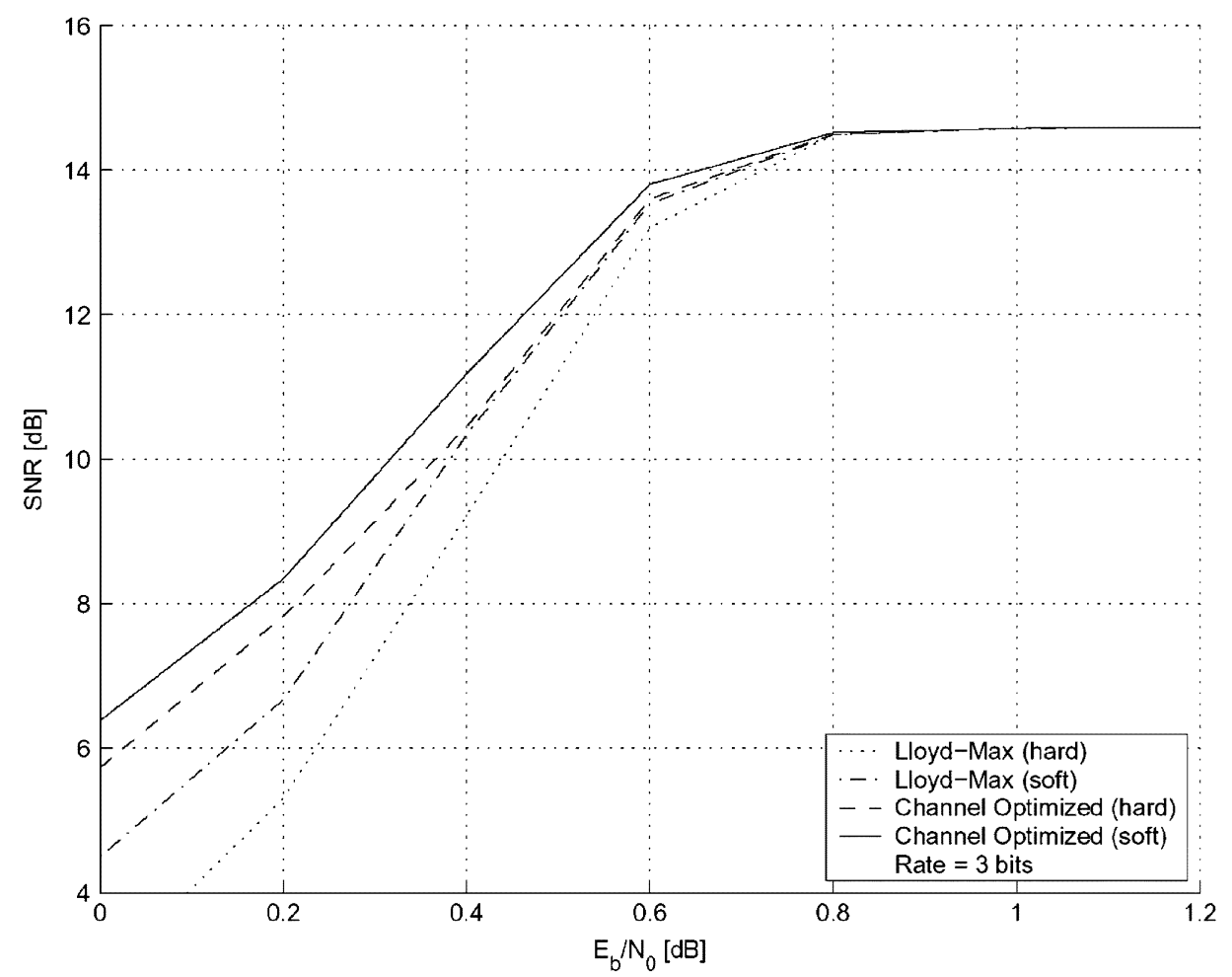

(a)

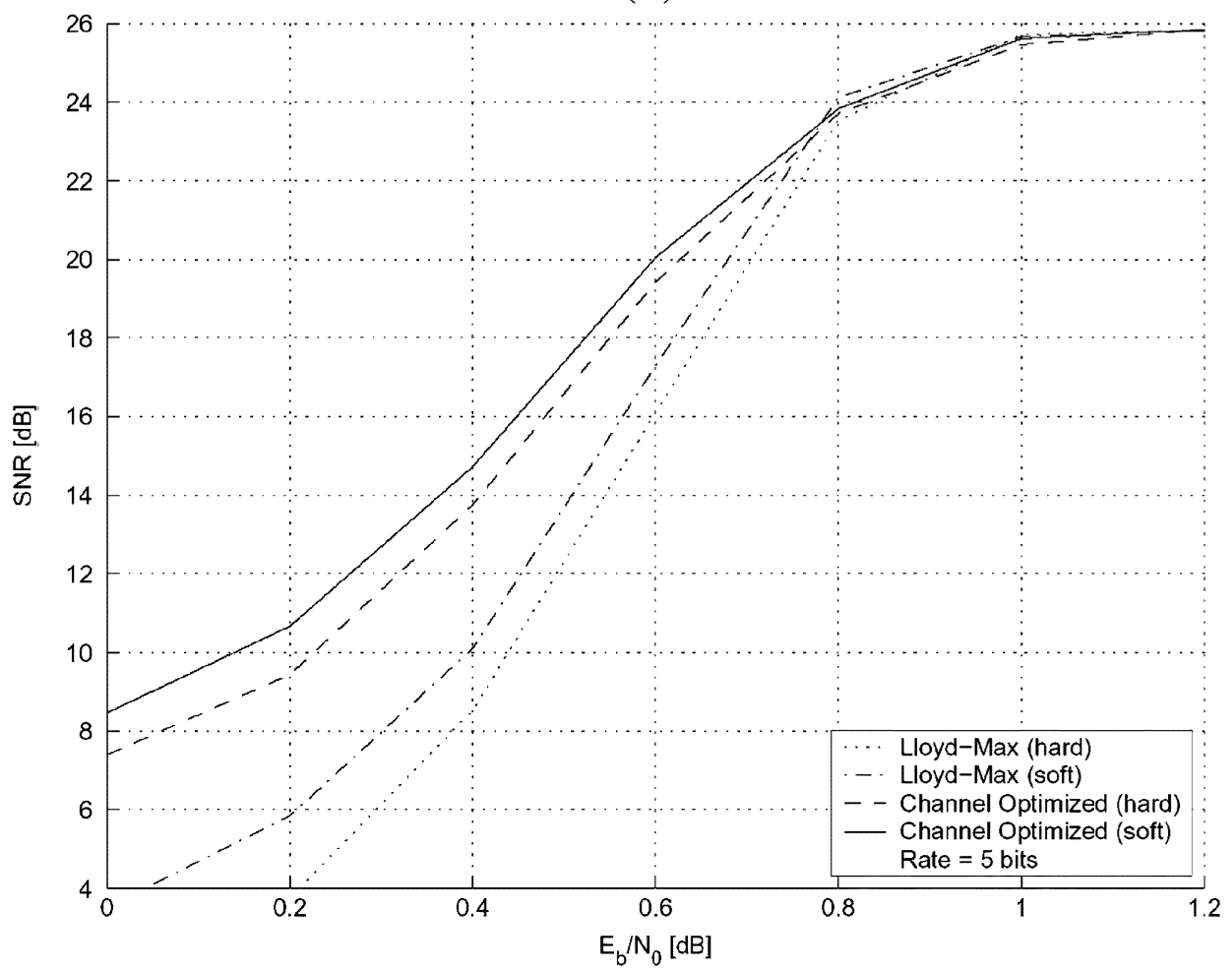

(b)

Fig. 4. SNR for 3- and 5-bit Lloyd-Max and channel-optimized quantizers using hard and soft reconstruction rules with rate $1 / 3$ turbo code with $M=2$ memory elements. (a) 3 bit quantizers, (b) 5 bit quantizers.

The soft-decision quantizer is designed using both the system of equations in (17) and the expected value in (33). During the design, the algorithm relies on the assumption that the bits within a code word are independent of each other. However, in practice, this assumption is not entirely valid. To improve the independence assumption, the entire block of 


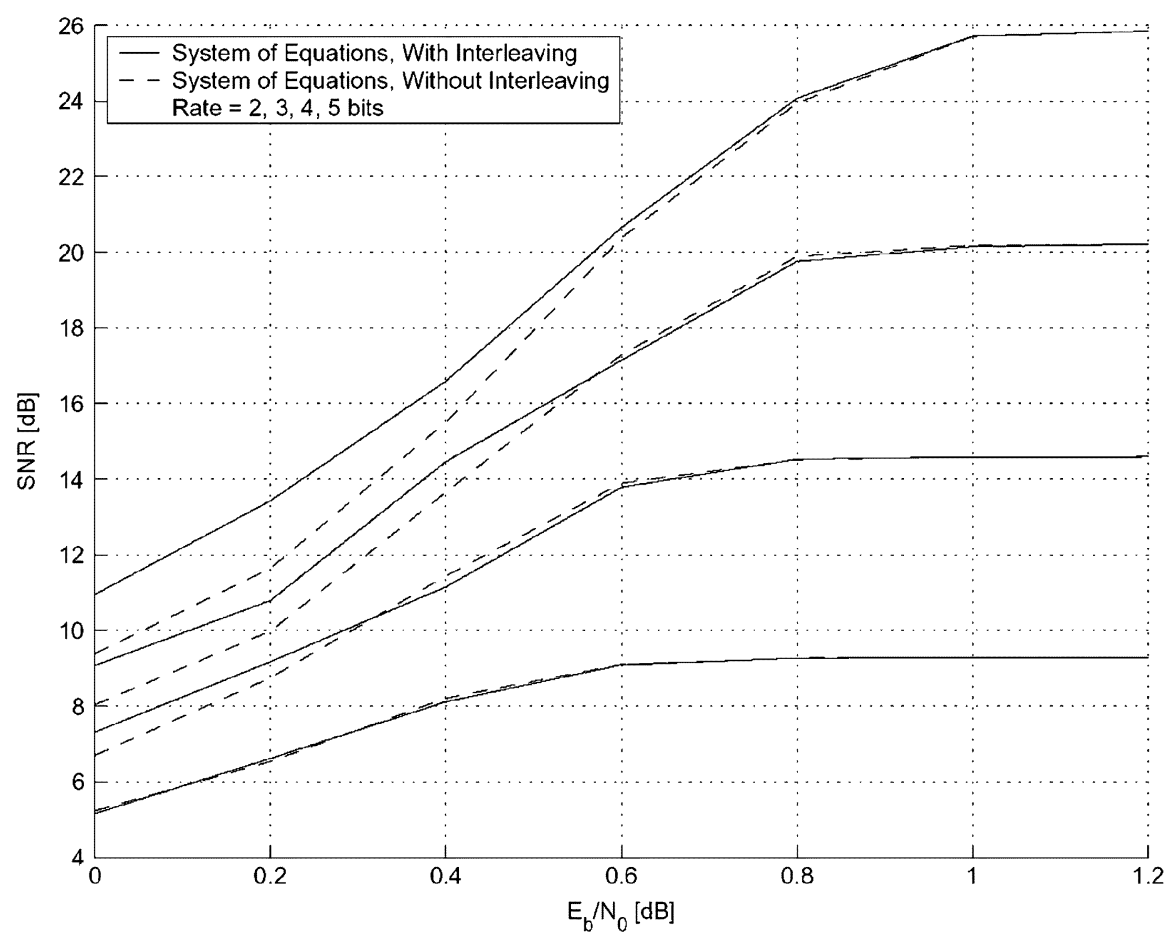

(a)

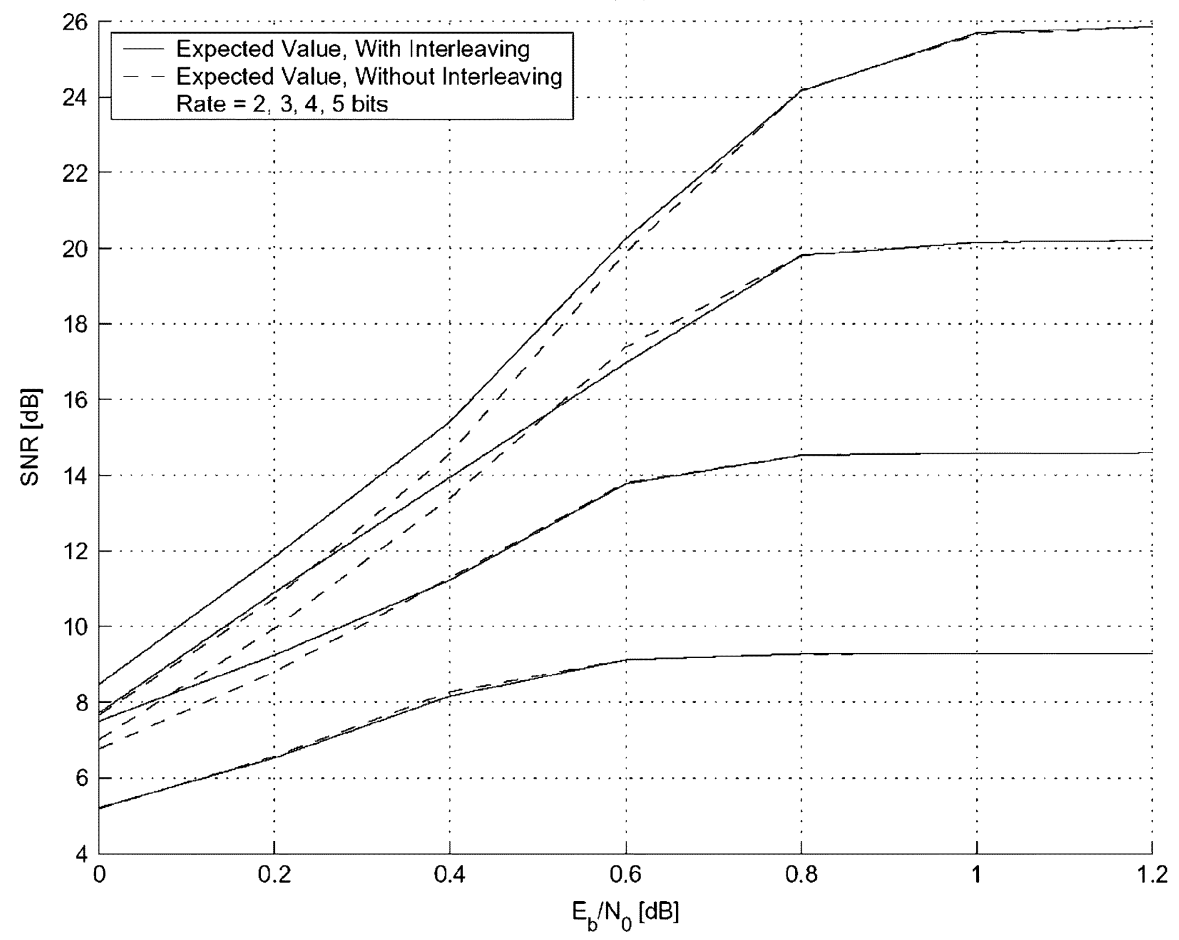

(b)

Fig. 5. Soft-decision quantizer with rate $1 / 3$ turbo code with $M=2$ memory elements, illustrating the effect of interleaving the quantized bits before channel coding. (a) Quantizer designed with system of equations. (b) Quantizer designed with expected value.

10000 bits is interleaved with an $S$-random interleaver before encoding with turbo code. Fig. 5 shows the effect of interleaving the bits before the channel code and an improvement in performance is achieved for both of the soft-decision quantizers. The results shown in Fig. 6 indicate that the quantizer designed with the system of equations offers an improvement over the expected value in both cases with and without interleaving of the bits.

\section{Channel Mismatch}

All the quantizers presented in this paper operate under the assumption that the channel noise level is known and does not 


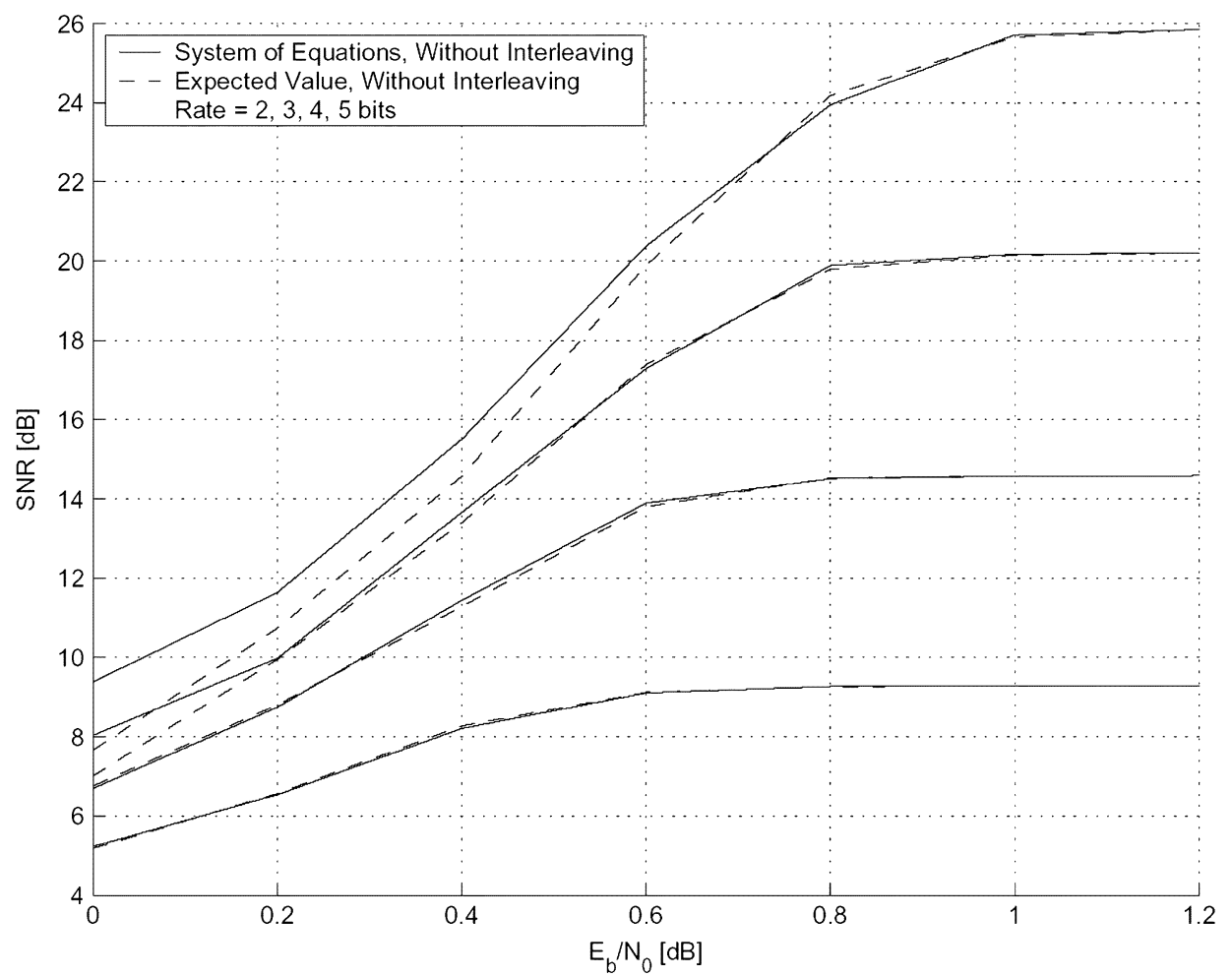

(a)

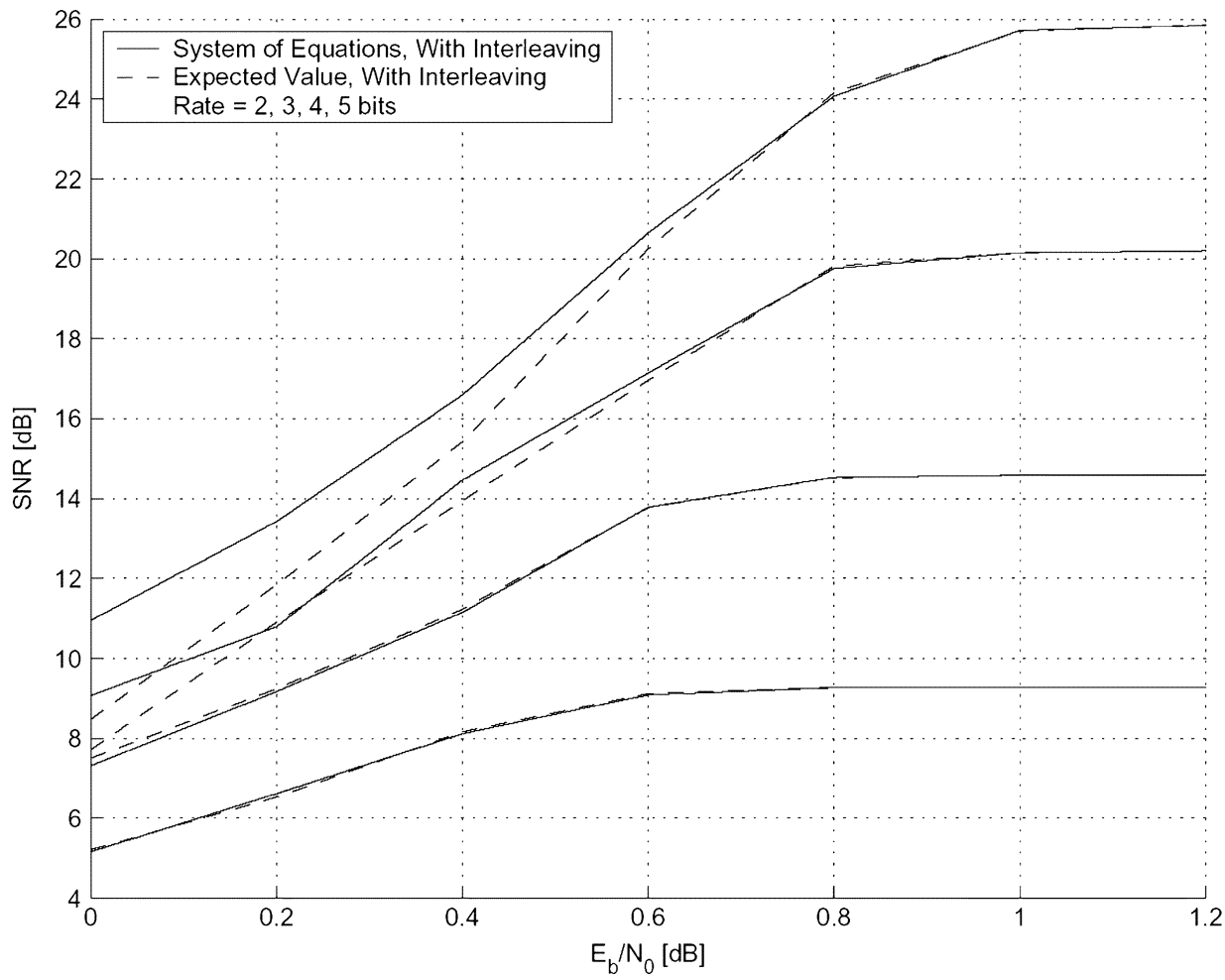

(b)

Fig. 6. Soft-decision quantizer with rate $1 / 3$ turbo code with $M=2$ designed with the system of equation and expected value methods. (a) Quantizer with no bit interleaving. (b) Quantizer with bit interleaving.

change during the transmission. In practice, the channel noise level fluctuates over time and Fig. 7 shows the effect of such mismatch for the 5-bit quantizers. The curve labeled fully optimized corresponds to the case that the quantizer and recon- structor are designed to match the operating channel-noise level. The curve labeled not optimized corresponds to the case that the quantizer is designed for $E_{b} / N_{0}=0.8 \mathrm{~dB}$ and operated at different channel noise levels. 


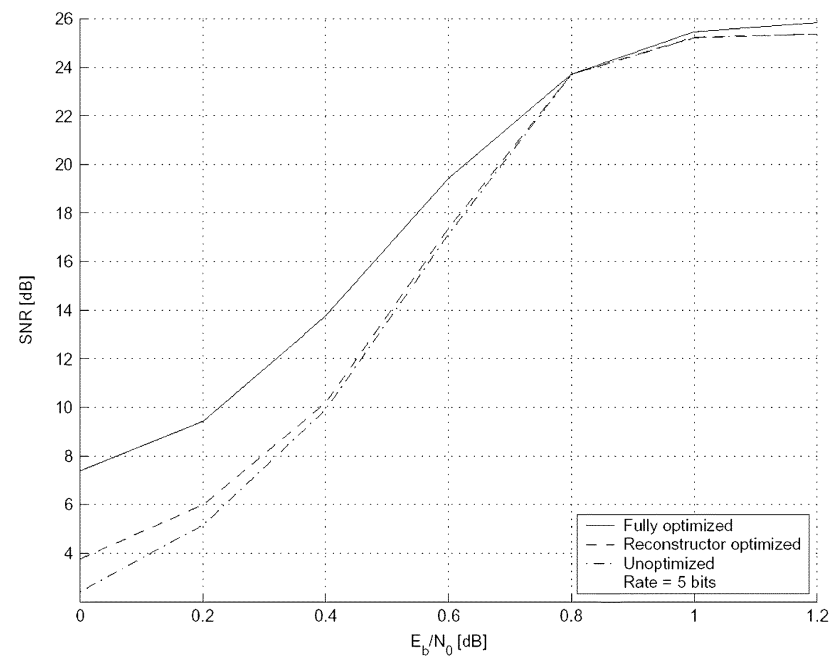

(a)

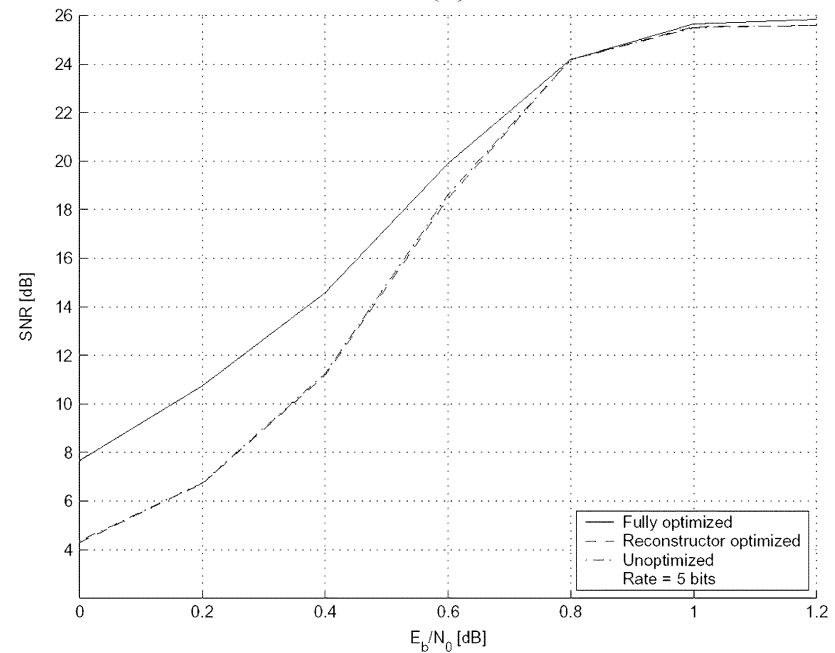

(b)

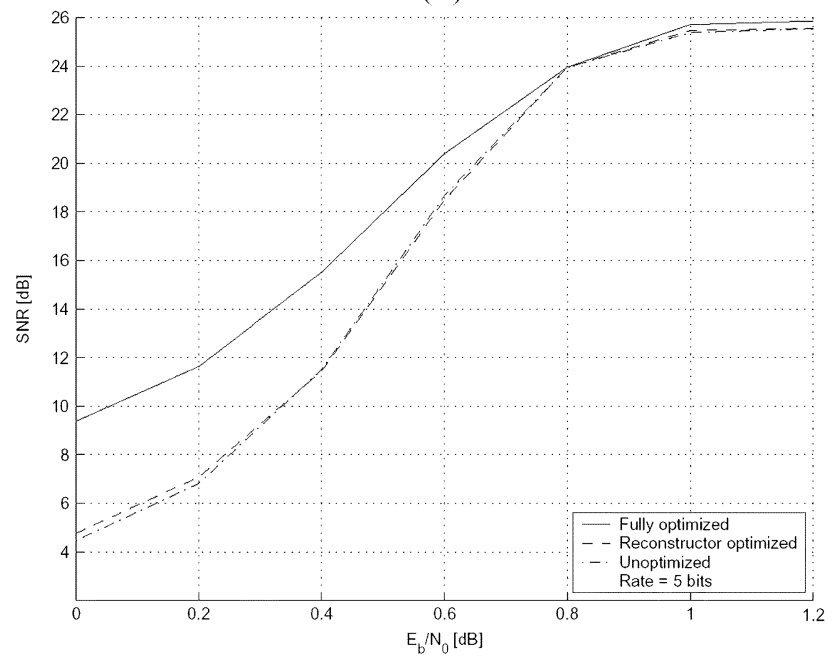

(c)

Fig. 7. Channel mismatch for the channel-optimized and soft-decision (expected value and system of equations) quantizers with rate 1/3 turbo code with $M=2$. (a) hard decision channel optimized. (b) Soft decision (expected value). (c) Soft decision (system of equations).

In another variation, the quantizer is designed for a fixed noise level; however, at the last step, the decoder is recalculated for the actual noise level, which results in a class of quantizers with the same quantizer and different reconstructors. This is done by designing the quantizer for $E_{b} / N_{0}=0.8 \mathrm{~dB}$ and at the last step the reconstructor is tuned to a particular value of $E_{b} / N_{0}$ using one of (10), (14), and (16) (depending on the system that is being designed) with the values of the parameters $\Theta_{i}(k)$ and $\Psi_{i}(j, k)$ that correspond to the (tuned to) value of the $E_{b} / N_{0}$. This situation is shown with the curve labeled reconstructor optimized. This situation is realistic because a soft-output channel decoder (including a turbo decoder) usually assumes knowledge of the channel noise variance and the reconstructor structure can be adjusted accordingly.

Fig. 7(a) shows the channel mismatch for the 5-bit channeloptimized quantizer with a rate $1 / 3$ turbo code. At $E_{b} / N_{0}=$ $0.2 \mathrm{~dB}$, the mismatch results in a $4.2-\mathrm{dB}$ degradation in the sample SNR from the fully optimized case and the adaptive reconstructor reduces this degradation to $3.4 \mathrm{~dB}$. Fig. 7(b) shows the channel mismatch for the 5-bit soft-decision quantizer designed with expected value. The sensitivity to the mismatch at $E_{b} / N_{0}=0.2 \mathrm{~dB}$ is $4.0 \mathrm{~dB}$; however, since the reconstructor design in (33) is independent of the noise level, no further gain can be achieved with reconstructor optimization. Fig. 7(c) shows the channel mismatch for the 5-bit soft-decision quantizer designed with the system of equations. The distortion at $E_{b} / N_{0}=0.2 \mathrm{~dB}$ is $4.5 \mathrm{~dB}$ below the fully optimized case; however, it is $0.3 \mathrm{~dB}$ better than the soft-decision quantizer designed with expected value. Optimizing the reconstructor further improves the distortion by $0.3 \mathrm{~dB}$.

\section{Quantization of Images}

To show the effect of the different design algorithms, we designed the quantizer to encode image pixels. The quantizers were designed using 18 images and tested using the $256 \times 256$ Lena image. Four different quantizers were tested, Lloyd-Max and channel-optimized quantizers with hard decision rule and the expected value and system of equations with the soft-decision rule.

Fig. 8 shows the SNR for the quantizer using 5-bit code words for different $E_{b} / N_{0}$ values. We see up to a $1-\mathrm{dB}$ improvement of the system of equation relative to expected value soft-decision and the channel-optimized quantizer. Fig. 10. shows the images for the four different quantizers encoded at 5 bits and transmitted over a channel with $E_{b} / N_{0}=0.2 \mathrm{~dB}$. Fig. 9 shows the Lena image encoded using discrete cosine transform using $4 \times 4$ blocks, where only the DC coefficients were encoded using 5-bit quantizers and transmitted over a channel with $E_{b} / N_{0}=0.4 \mathrm{~dB}$.

Figs. 9(a) and 10(a) show the Lloyd-Max quantized image and we see that the images have a significant salt-and-pepper noise present. Figs. 9(b) and 10(b) show the quantizer with binary symmetric channel. The images show that the salt-andpepper noise is still present, but is reduced. Figs. 9(c) and 10(c) show the soft quantizer with the reconstruction levels calculated as the expected value. The amount of noise is reduced compared to the Lloyd-Max quantizer. Figs. 9(d) and 10(d) show the soft quantizer with the reconstruction levels. These images show the least amount of noise present. Therefore, the soft reconstruction quantizer with system of equations seems to be the best suited for image coding. 


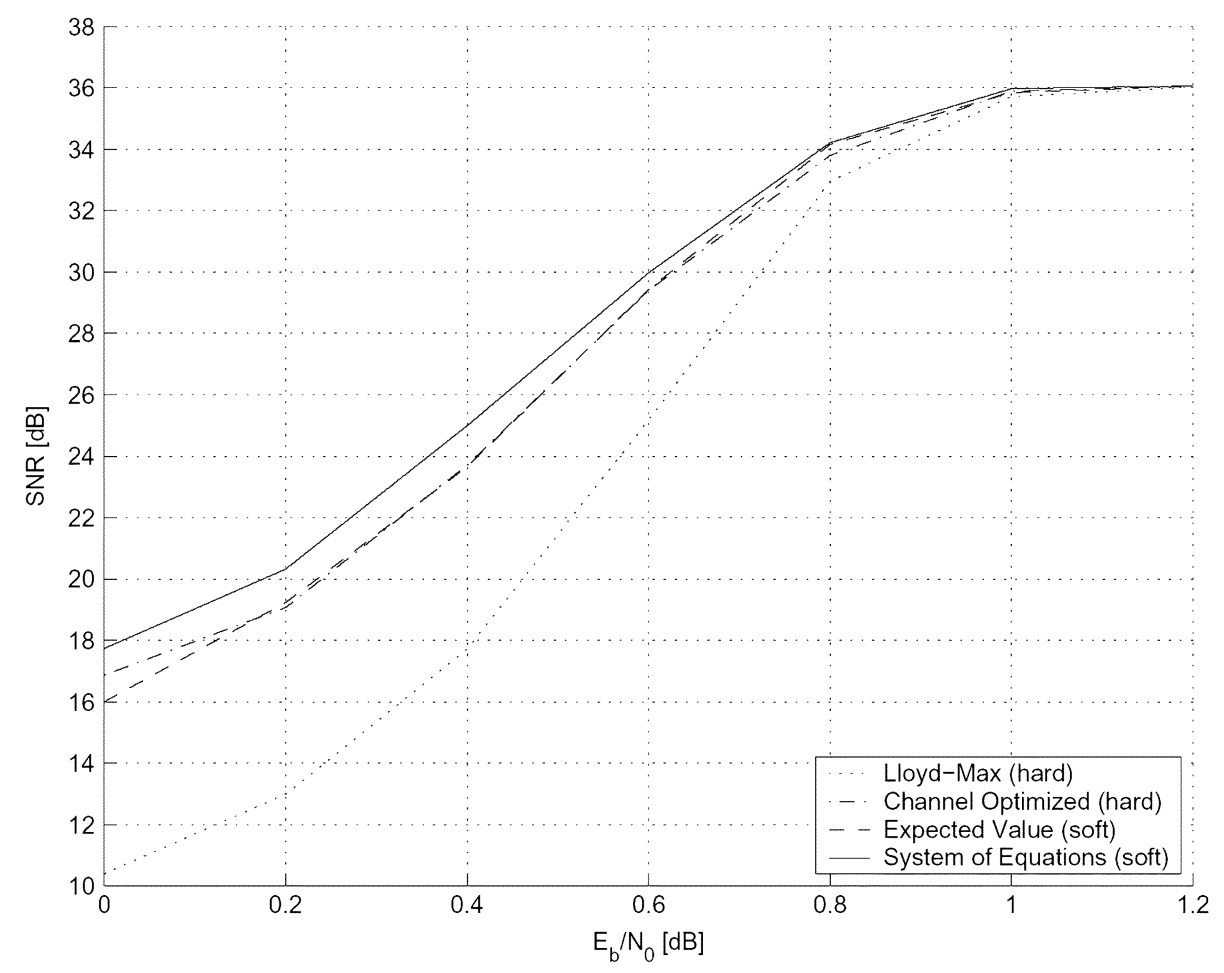

Fig. 8. SNR for the $256 \times 256$ Lena image quantized with 5 -bit quantizers.

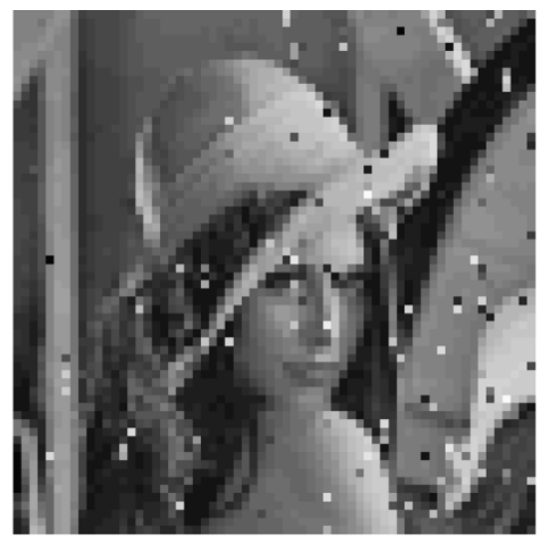

(a)

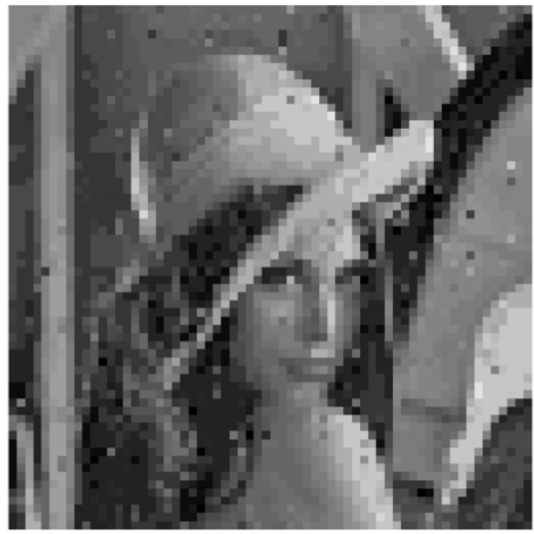

(c)

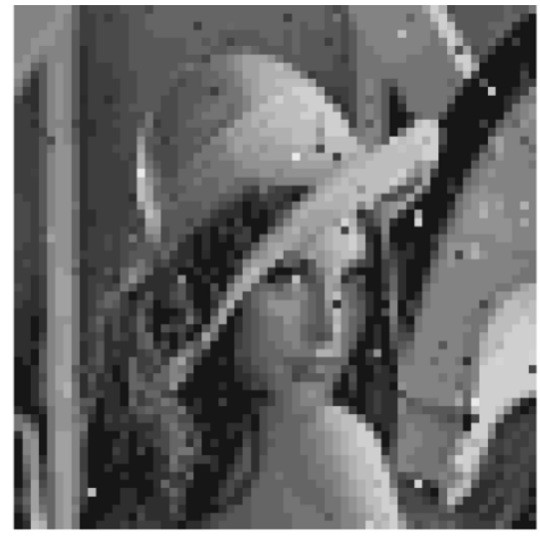

(b)

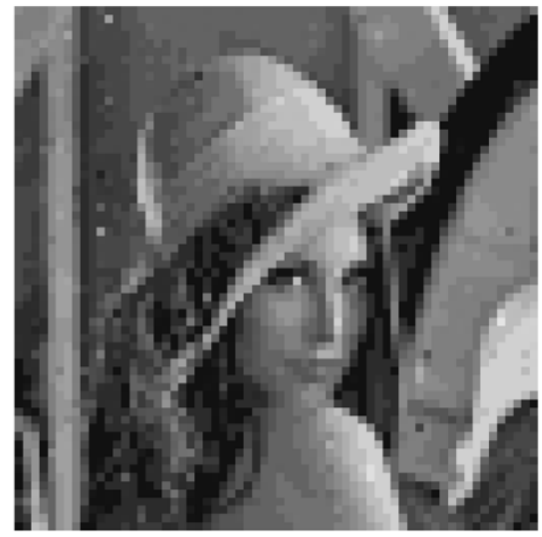

(d)

Fig. 9. Quantization and coding of the $256 \times 256$ Lena image with only the DC coefficients of the DCT transform with $4 \times 4$ blocks using a 5 -bit quantizer with the channel noise of $E_{b} / N_{0}=0.4 \mathrm{~dB}$. (a) Lloyd-Max algorithm. (b) Binary symmetric. (c) Soft (expectation). (d) Soft (system of equation). 


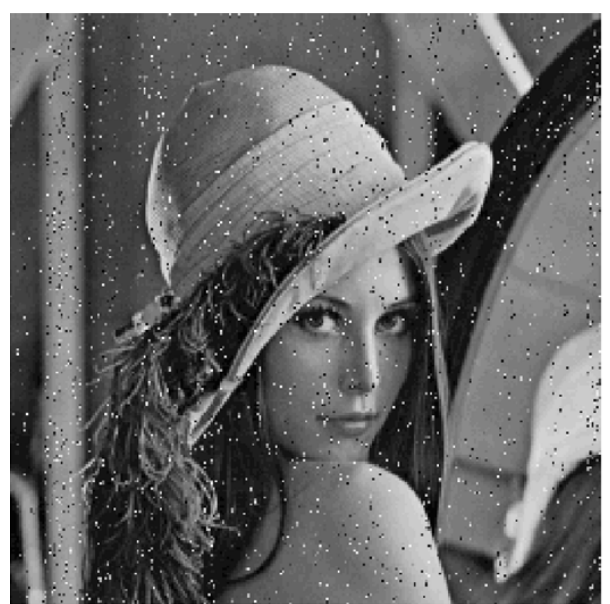

(a)

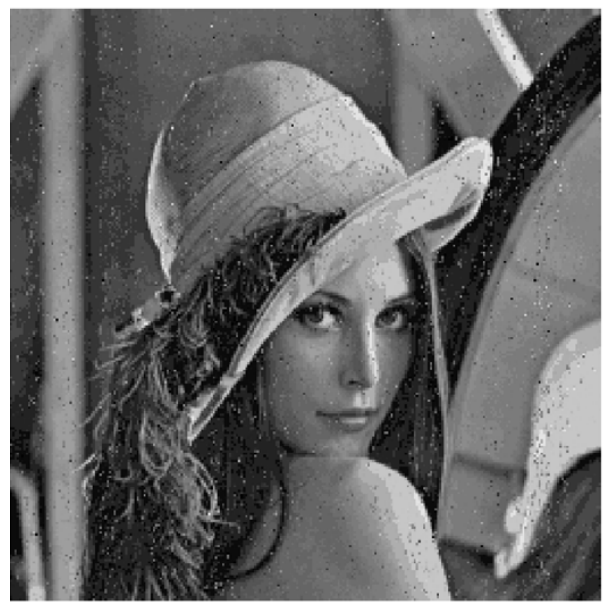

(c)

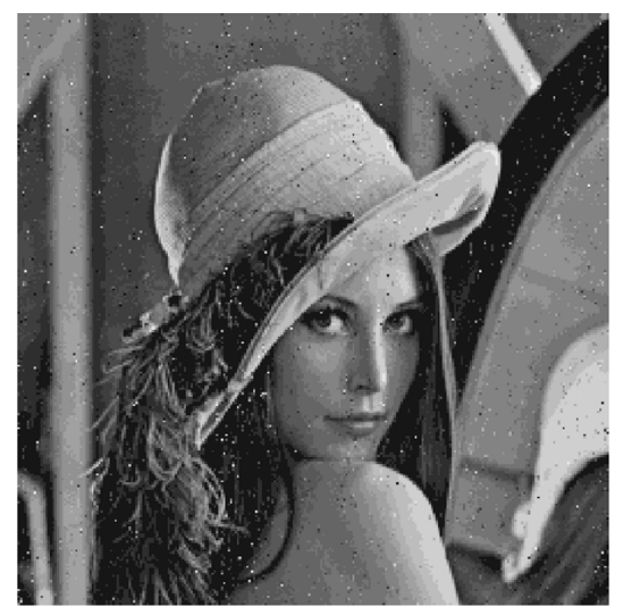

(b)

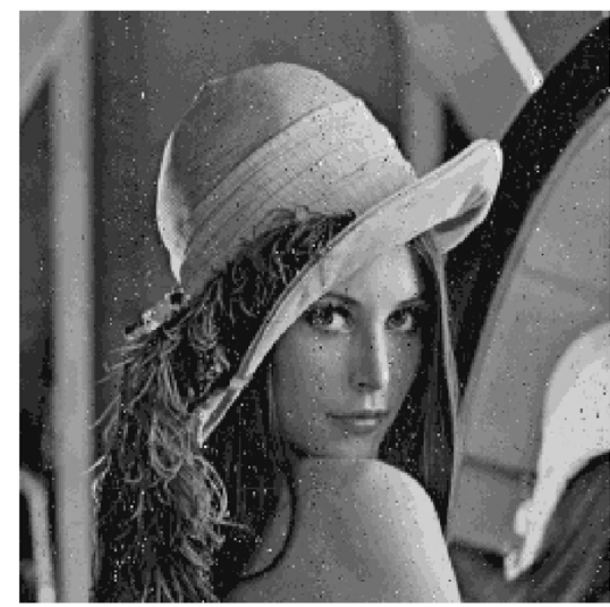

(d)

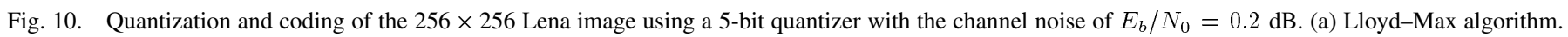
(b) Binary symmetric. (c) Soft (expectation). (d) Soft (system of equation).

\section{SUMMARY}

We have discussed a new method of combined sourcechannel coding using turbo codes or, more generally, a channel code with reliability information. A system is presented that transmits a discrete time, continuous amplitude signal over a noisy channel. The transmitted samples are encoded using a fixed rate scalar quantizer and turbo-code channel coding is used for error correction. The system performance is improved by using a soft reconstruction of samples using the reliability information available at the channel decoder. The performance of the presented quantizers has been shown to be up to $1.0 \mathrm{~dB}$ better than the channel optimized scalar quantizer. The effect of the tradeoff between the rates allocated to the source and channel coders, as well as the effect of the channel mismatch, are studied. It is shown that the increase in distortion due to a channel mismatch can be reduced from approximately $3.5 \mathrm{~dB}$ to approximately $1.5 \mathrm{~dB}$ with an adaptive receiver.

\section{APPENDIX}

Consider the definition of the overall distortion given in (6). This average distortion is minimized by setting its derivative with respect to $R_{k}, k=1, \ldots, N$, equal to zero. This results in $N$ equations in terms of the $N$ reconstruction levels

$$
2 \sum_{i=1}^{N} \int_{S_{i}} x P(x) d x\left[\frac{d B_{i}}{d R_{k}}\right]=\sum_{i=1}^{N} \int_{S_{i}} P(x) d x\left[\frac{d C_{i}}{d R_{k}}\right]
$$

where $k=1, \ldots, N$. To optimize the system for a soft-decision quantizer with reconstruction function given in (11), we substitute $B_{i}$ and $C_{i}$ defined in (4) to obtain a set of linear equations in $(17)$, restated here as

$$
\sum_{i=1}^{N} \Theta_{i}(k) \int_{S_{i}} x P(x) d x=\sum_{j=1}^{N} R_{j} \sum_{i=1}^{N} \Psi_{i}(j, k) \int_{S_{i}} P(x) d x
$$

where $k=1, \ldots, N$. By a direct replacement of (13) into (31) and using the identity

$$
\begin{aligned}
& P\left(\mathbf{v} \mid \mathbf{u}=\mathbf{u}_{i}\right)=\frac{P\left(\mathbf{u}=\mathbf{u}_{i} \mid \mathbf{v}\right)}{P\left(\mathbf{u}=\mathbf{u}_{i}\right)} P(\mathbf{v}), \\
& \text { for } P\left(\mathbf{u}=\mathbf{u}_{i}\right) \neq 0
\end{aligned}
$$

the set of (17) simplifies to the trivial case where the reconstruction level $R_{i}$ is equal to expected value of the source samples in 
the corresponding partition $S_{i}$, i.e., $R_{i}=E\left(x \mid x \in S_{i}\right)$. However, this requires all the partitions to be nonempty in order to use the identity in (32), i.e.,

$$
R_{i}=\frac{\int_{S_{i}} x P(x) d x}{\int_{S_{i}} P(x) d x}, \quad \text { if } \int_{S_{i}} P(x) d x>0, \quad \forall i .
$$

As the channel noise increases, some of the quantizer partitions become empty. In mathematical terms, the $m$ th partition will be empty if for all inputs $x$, we have

$$
\forall x \quad \exists i \neq m, \quad \text { such that } D_{i}(x)<D_{m}(x)
$$

where $D_{i}(x), D_{m}(x)$ are given in (2). Note that such empty partitions are decided by the design algorithm to optimize the overall performance and should not be confused with the cases of having empty partitions due to using a small training data sequence.

This situation arises because the known turbo-decoding algorithms are based on multiplying the probability of the bits to compute the probability of the symbols (note that the iterative turbodecoding operation is heavily dependent on this independence assumption). Due to this feature, the reconstruction levels should be optimized in each step of the iterative design algorithm, taking into account that some of the partitions may be empty. The presented system of equations computes these reconstruction levels $\left(R_{i}\right.$ 's) in an optimum manner for the given iteration.

\section{ACKNOWLEDGMENT}

The authors would like to thank Associate Editor J. Shea and the anonymous reviewers for their detailed comments, which have significantly helped to improve the quality of this paper.

\section{REFERENCES}

[1] J. Max, "Quantizing for minimum distortion," IEEE Trans. Inform. Theory, vol. IT-6, no. 2, pp. 7-12, Mar. 1960.

[2] S. P. Lloyd, "Least squares quantization in PCM," IEEE Trans. Inform. Theory, vol. IT-28, no. 2, pp. 129-137, Mar. 1982.

[3] Y. Linde, A. Buzo, and R. M. Gray, "An algorithm for vector quantizer design," IEEE Trans. Commun., vol. COM-28, no. 1, pp. 84-94, Jan. 1980.

[4] A. J. Kurntenbach and P. A. Wintz, "Quantizing for noisy channels," IEEE Trans. Commun. Technol., vol. COM-17, no. 2, pp. 291-302, Apr. 1969.

[5] N. Farvardin and V. A. Vaishampayan, "Optimal quantizer design for noisy channels: An approach to combined source-channel coding," IEEE Trans. Inform. Theory, vol. 33, no. IT-6, pp. 827-838, Nov. 1987.

[6] N. Farvardin, "A study of vector quantization for noisy channels," IEEE Trans. Inform. Theory, vol. 36, no. 4, pp. 799-809, Jul. 1990.

[7] N. Farvardin and V. A. Vaishampayan, "On the performance and complexity of channel-optimized vector quantizers," IEEE Trans. Inform. Theory, vol. 37, no. 1, pp. 155-160, Jan. 1991.

[8] V. A. Vaishampayan and N. Farvardin, "Joint design of block source codes and modulation signal sets," IEEE Trans. Inform. Theory, vol. 38, no. 4, pp. 1230-1248, Jul. 1992.

[9] F. I. Alajaji and N. C. Phamdo, "Soft-decision COVQ for Rayleighfading channels," IEEE Commun. Lett., vol. 2, no. 6, pp. 162-164, Jun. 1998.

[10] N. Phamdo and F. Alajaji, "Soft-decision demodulation design for COVQ over white, colored, and ISI Gaussian channels," IEEE Trans. Commun., vol. 48, no. 9, pp. 1499-1506, Sep. 2000.

[11] G.-C. Zhu and F. I. Alajaji, "Soft-decision COVQ for turbo-coded AWGN and Rayleigh fading channels," IEEE Commun. Lett., vol. 5, no. 6, pp. 257-259, Jun. 2001

[12] M. Skoglund and P. Hedelin, "Vector quantization over a noisy channel using soft decision decoding," in Proc. IEEE Int. Conf. Acoustics, Speech, and Signal Processing, Adelaide, Australia, Apr. 1994, pp. 605-608.
[13] — "Hadamard-based soft decoding for vector quantization over noisy channels," IEEE Trans. Inform. Theory, vol. 45, no. 2, pp. 515-532, Mar 1999.

[14] M. Skoglund, "A soft decoder vector quantizer for a Rayleigh fading channel-Application to image transmission," in Proc. IEEE Int. Conf. Acoustics, Speech, and Signal Processing, Detroit, MI, May 1995, pp. 2507-2510.

[15] T. Ottosson and M. Skoglund, "Soft multiuser decoding for vector quantization over Rayleigh fading CDMA channels," in Proc. IEEE Symp. Spread Spectrum Techniques and Applications, Mainz, Germany, Sep. 1996, pp. $22-25$

[16] - "Soft multiuser decoding for vector quantization over a CDMA channel," IEEE Trans. Inform. Theory, vol. 46, no. 3, pp. 327-337, Mar 1998.

[17] D. Miller and K. Rose, "Combined source-channel vector quantization using deterministic annealing," IEEE Trans. Inform. Theory, vol. 38, no. 7, pp. 1249-1257, Jul. 1994

[18] S. Gadkari and K. Rose, "Robust vector quantizer design by noisy channel relaxation," IEEE Trans. Inform. Theory, vol. 47, no. 8, pp 1113-1116, Aug. 1999.

[19] K. Rose, "Deterministic annealing for clustering, compression, classification, regression and related optimization problems," Proc. IEEE, vol. 86, no. 11 , pp. 2210-2239, Nov. 1998.

[20] K.-P. Ho, "Soft-decoding vector quantizer using reliability information from turbo codes," IEEE Commun. Lett., vol. 3, no. 7, pp. 208-210, Jul. 1999.

[21] J. He, D. J. Costello, Y. Huang, and R. L. Stevenson, "On the application of turbo codes to the robust transmission of compressed images," in IEEE Int. Conf. Image Processing, Santa Barbara, CA, Oct. 1997, pp. 559-562.

[22] Z. Peng, Y. Huang, D. J. Costello, and R. L. Stevenson, "On the application of turbo codes to the robust transmission of compressed images," in Proc. Conf. Information Sciences and Systems, Princeton, NJ, Mar 1998, pp. 330-335.

[23] W. B. Pennerbaker and J. L. Mithchell, JPEG: Still Image Compression Standard. New York: Van Nostrand Rheinhold, 1993.

[24] L. R. Bahl, J. Cocke, F. Jelinek, and J. Raviv, "Optimal decoding of linear codes for minimizing symbol error rate," IEEE Trans. Inform. Theory, vol. 20, no. IT-2, pp. 284-287, Mar. 1974.

[25] C. Berrou, A. Glavieux, and P. Thitimajshima, "Near Shannon limit errorcorrecting coding and decoding: Turbo codes(1)," in Proc. 1993 Int. Conf. Communication, Geneva, Switzerland, May 1993, pp. 1064-1070.

[26] J. Bakus and A. K. Khandani, "Combined source-channel coding using turbo-codes," in Proc. Conf. Information Sciences and Systems, Baltimore, MD, Mar. 1997, pp. 309-313.

[27] —_ "Combined source-channel coding using turbo-codes," Electron. Lett., vol. 33, no. 13, pp. 1613-1614, Sep. 1997.

[28] J. Hagenauer, E. Offer, and L. Papke, "Iterative decoding of binary block and conventional codes," IEEE Trans. Inform. Theory, vol. 42, no. 2, pp. 429-445, Mar. 1996.

[29] S. Dolinar and D. Divsalar, "Weight distributions for turbo codes using random and nonrandom permutations," Jet Propulsion Lab., Pasadena, CA, Tech. Rep., Aug. 1995.

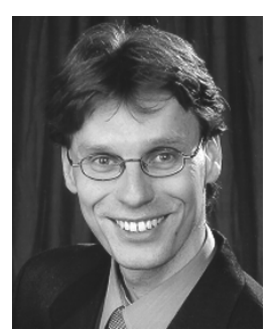

Jan Bakus received the B.A.Sc. and M.A.Sc. degrees in electrical engineering from the University of Waterloo, Waterloo, ON, Canada, in 1996 and 1998, respectively, where he is currently working toward the $\mathrm{Ph} . \mathrm{D}$. degree in machine learning.

$\mathrm{He}$ is currently with Maplesoft, Waterloo, ON, Canada, as an Applications Engineer, where he is responsible for the development of applications for the Maple scientific computing software.

Mr. Bakus is the Recipient of the Carl Pollock Fellowship Award from the University of Waterloo and the Datatel Scholars Foundation scholarship from Datatel Scholars Foundation, Fairfax, VA.

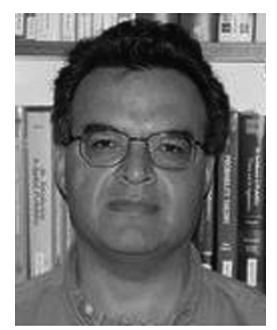

Amir K. Khandani (M'93) received the B.A.Sc. and M.A.Sc. degrees from the University of Tehran, Tehran, Iran, and the Ph.D. degree from McGill University, Montreal, QC, Canada, in 1985 and 1992, respectively.

He was a Research Associate with INRS-Telecommunications, Montreal, QC, Canada, for one year. In 1993, he joined the Department of Electrical and Computer Engineering, University of Waterloo, Waterloo, ON, Canada, where he currently is a Professor. 